\title{
Acute toxicity and early cosmetic outcome in patients treated with multilumen balloon brachytherapy with skin spacing $\leq 7.0$ millimeters
}

\author{
Kiwhoon Lee, MD', Amy Quillo, MD', Dustin Dillon, MD', Keith Sowards, MD', Allison Hatmaker, MD², \\ Anthony Dragun, MD, Ass. Prof
}

University of Lovisville School of Medicine, James Graham Brown Cancer Center, ${ }^{2}$ Baptist East Hospital, Lovisville, KY, USA

\begin{abstract}
Purpose: To review institutional experience treating patients who underwent breast conserving surgery and adjuvant accelerated partial breast irradiation with multilumen balloon brachytherapy (MLB) with close skin spacing $(\leq 7 \mathrm{~mm})$.

Material and methods: Since July 2009, 26 patients with skin spacing $\leq 7.0 \mathrm{~mm}$ were treated with breast-conserving therapy and adjuvant MLB brachytherapy. Patients were treated with either the Contura or MammoSite ML catheter to a total dose of 34 Gy in 10 fractions. Patients were assessed for acute toxicity at the completion of treatment and 1-month post treatment. Cosmesis and late toxicity were assessed at three-month intervals thereafter.

Results: The median age of the patients was 56 years and median follow-up was 9 months. Sixteen patients had skin spacing of 5.0-7.0 mm, 10 with $<5.0 \mathrm{~mm}$ (median 5.8). The median percentage of the target (PTV_EVAL) receiving $\geq 95 \%$ of the prescription dose was $95.6 \%$. The median volume of PTV_EVAL receiving $\geq 200 \%$ of the prescription dose was 6.1 cc. The maximum skin dose was $118.2 \%$ (median). The most commonly observed acute toxicity was grade $1-2$ dermatitis $(65.4 \%)$. The rate of post-treatment seroma and infection was $38.5 \%$ and $3.8 \%$, respectively. Excellent/good cosmetic outcomes seen at the time of last follow-up was $92.3 \%$.

Conclusions: MLB brachytherapy is safe and feasible in patients with close skin spacing, with acute toxicity and early cosmesis similar to other published series. These devices may broaden the application of balloon brachytherapy in patients previously excluded from this treatment based on anatomy.

J Contemp Brachyther 2012; 4, 1: 8-13 DOI: 10.5114/jcb.20l2.27946
\end{abstract}

Key words: brachytherapy, APBI, Contura, MammoSite, Multi-catheter.

\section{Purpose}

Accelerated partial breast irradiation (APBI) has become an acceptable treatment option in selected patients that have undergone breast conservation therapy [1]. Based on several retrospective series and randomized data, acceptable 5 and 10 year local control rates along with good or excellent cosmetic outcomes have been shown [2-4]. In the American Society of Breast Surgeons Mammosite Breast Brachytherapy Registry Trial, the 2 year ipsilateral breast tumor recurrence rate was $1 \%$, while reporting good to excellent cosmetic outcomes in $93 \%$ of patients at 3 years [5]. Thus far, the largest cohort of patients treated with APBI has been with catheter based interstitial brachytherapy (IB). This group has also provided the longest follow up data. IB, despite its proven track record, has some disadvantages, which include its complexity and reproducibility that may limit the availability of this technique. The development of the Mammosite applicator (HologicInc, Bedford, MA) allowed for a more simple and streamlined method to deliver APBI.

With the advent of the single lumen Mammosite, balloon surface to skin distances of $>7 \mathrm{~mm}$ is generally recommended to avoid excessive skin dose and decrease the probability of suboptimal cosmetic outcomes, including the ongoing NSABP B-39 trial. One of the limitations with the original Mammosite is that there is reduced ability to manipulate dose because of a single lumen design. There have been new intracavitary brachytherapy devices released recently that may help address some of these issues. MultiLumen Balloon (MLB) brachytherapy catheters such as the Mammosite ML and Contura (SenoRx, Inc., Irvine, CA) have recently been introduced in 2007 and 2009, respectively. The additional lumens that are present in these catheters may make dose shaping possible to provide for increased 
target volume coverage while sparing normal tissue and avoiding compromise of other dosimetric parameters. There have been several dosimetric studies that have presented advantages with the use of MLB brachytherapy and have shown that more stringent dosimetric goals are able to be met [6-8]. In comparison to the single-lumen MammoSite, it appears that MLB brachytherapy catheters provided significant improvements in dosimetric endpoints such as maximum skin dose and chest wall dose [8]. This was despite closer skin spacing in patients that were treated with MLB brachytherapy. Even in patients with limited skin spacing (3-6 mm), the Contura catheter did not need to be explanted due to concerns regarding maximum skin dose [9].

The purpose of this study was to: 1 . examine established dosimetric parameters in a specific subset of patients with limited skin spacing treated with multi-lumen balloon brachytherapy and 2. to analyze acute toxicity and early cosmetic outcome in patients treated with skin spacing $\leq 7 \mathrm{~mm}$.

\section{Material and methods}

Dosimetric and acute toxicity endpoints were evaluated in 26 consecutive patients undergoing MLB brachytherapy with skin spacing $\leq 7 \mathrm{~mm}$ as adjuvant treatment in breast conservation therapy. These patients were treated consecutively from July 14, 2009 to August 10, 2010. The patients had at least 3 months of follow up. This study was approved by the University of Louisville institutional review board.

\section{Inclusion/exclusion criteria}

All 26 patients underwent lumpectomy with negative surgical margins after a histologically confirmed diagnosis of ductal carcinoma in situ and/or invasive carcinoma. All patients were older than 50 years of age. Patients with invasive carcinoma underwent an axillary staging procedure which confirmed axillary lymph nodes to be pathologically negative. All tumors were $\leq 3 \mathrm{~cm}$ in maximum diameter. No patients were classified as "unsuitable" according to the ASTRO consensus statement on APBI [1]. Patient had skin spacing $\leq 7 \mathrm{~mm}$ which was measured from the balloon surface to the skin.

\section{Technical and treatment guidelines}

The balloon catheters were placed either using an open cavity technique or an ultrasound guided technique by the surgeon. Prophylactic antibiotics were prescribed while the catheter was in place. The patients were brought to the Radiation Oncology Department for CT planning. The total prescribed dose of $34 \mathrm{~Gy}$ was delivered to the planning target volume for evaluation (PTV_EVAL) divided in 10 fractions over 5 consecutive treatment days. The treatment fractions were delivered at least 6 hours apart. The balloon's position and inflation volume were verified before each fraction with ultrasound. Proper orientation was also confirmed to be in accordance with that at initial CT planning by aligning the skin mark to the line on the shaft of the brachytherapy device. Treatment was delivered using Varian (Palo Alto, CA) VariSource HDR remote afterloader.

The PTV_EVAL was defined as the breast tissue immediately surrounding the balloon to a $10 \mathrm{~mm}$ distance from the balloon surface. PTV_EVAL excluded the volume
$5 \mathrm{~mm}$ from the skin surface and also the chest wall/pectoralis muscles. When determining coverage of PTV_EVAL, the volume of air/fluid trapped outside the balloon was accounted for as it displaces a percentage of the target beyond $1 \mathrm{~cm}$ from the balloon surface. The trapped air/fluid was contoured and the total volume obtained. The percentage of PTV_EVAL displaced was then calculated. When determining PTV_EVAL dose coverage, the displaced percentage was subtracted. The avoidance structure designated as "SKIN" was defined as a volume extending from the surface of the breast to a $5 \mathrm{~mm}$ depth, encompassing the quadrant overlying the lumpectomy cavity.

Dose volume histogram analysis was performed as per the NSABP B-39 [10]. Target volume coverage of $\geq 90 \%$ of the prescribed dose covered $\geq 90 \%$ of PTV_EVAL (accounting for volume of trapped air/fluid). If possible, the maximum skin dose was reduced to low as achievable without sacrificing coverage of the target volume. The maximum skin dose was limited to $\leq 145 \%$. The volume of breast tissue receiving $150 \%$ of prescribed dose was limited to $\leq 50 \mathrm{cc}$. The volume of breast tissue receiving $200 \%$ of prescribed dose was limited to $\leq 10 \mathrm{cc}$. A sample treatment plan for a typical patient included in this analysis is shown in Fig. 1.

\section{Cosmesis and toxicity evaluations}

Patients were followed one month after completion of brachytherapy, then at 3 month intervals by the surgeon and radiation oncologist. Acute toxicity was graded according to the most severe reaction observed. Baseline mammography was performed approximately 12 months after completion of treatment. Follow up was complete through November 1, 2010.

Infections were defined as any evidence of mastitis, cellulitis or abscess that was observed and required intervention (oral antibiotics, intravenous antibiotics or surgical intervention). Examination of the skin was performed at every follow up. Acute skin reactions were divided into four categories: none, erythema, dry desquamation and moist desquamation. The presence or absence of telangiectasia was documented at each follow up. Toxicity was graded as none, $<2 \mathrm{~cm}^{2}$ and scant, $<2 \mathrm{~cm}^{2}$ and dense, and $>2 \mathrm{~cm}^{2}$. The level of pain was divided into 5 categories: none, intermittent, persistent, medication required and surgical intervention. Evaluation for subcutaneous toxicity was performed at each follow up by physical examination or with ultrasound. Subcutaneous toxicity was divided into palpable, visible and those requiring surgery. Cosmetic outcome was evaluated using the Harvard criteria. An excellent cosmetic outcome was defined as the treated breast nearly identical to the untreated breast. A good cosmetic outcome was minimal radiation effects of the treated breast. A fair score was given when radiation effects were readily observable and significant. A poor score was used when there was severe distortion of the treated breast due to radiation effects.

\section{Results}

A total of 26 patients were treated with MLB brachytherapy with skin spacing $\leq 7 \mathrm{~mm}$. Median follow up was 9 months (range 3-15.8). The patient and tumor characteri- 


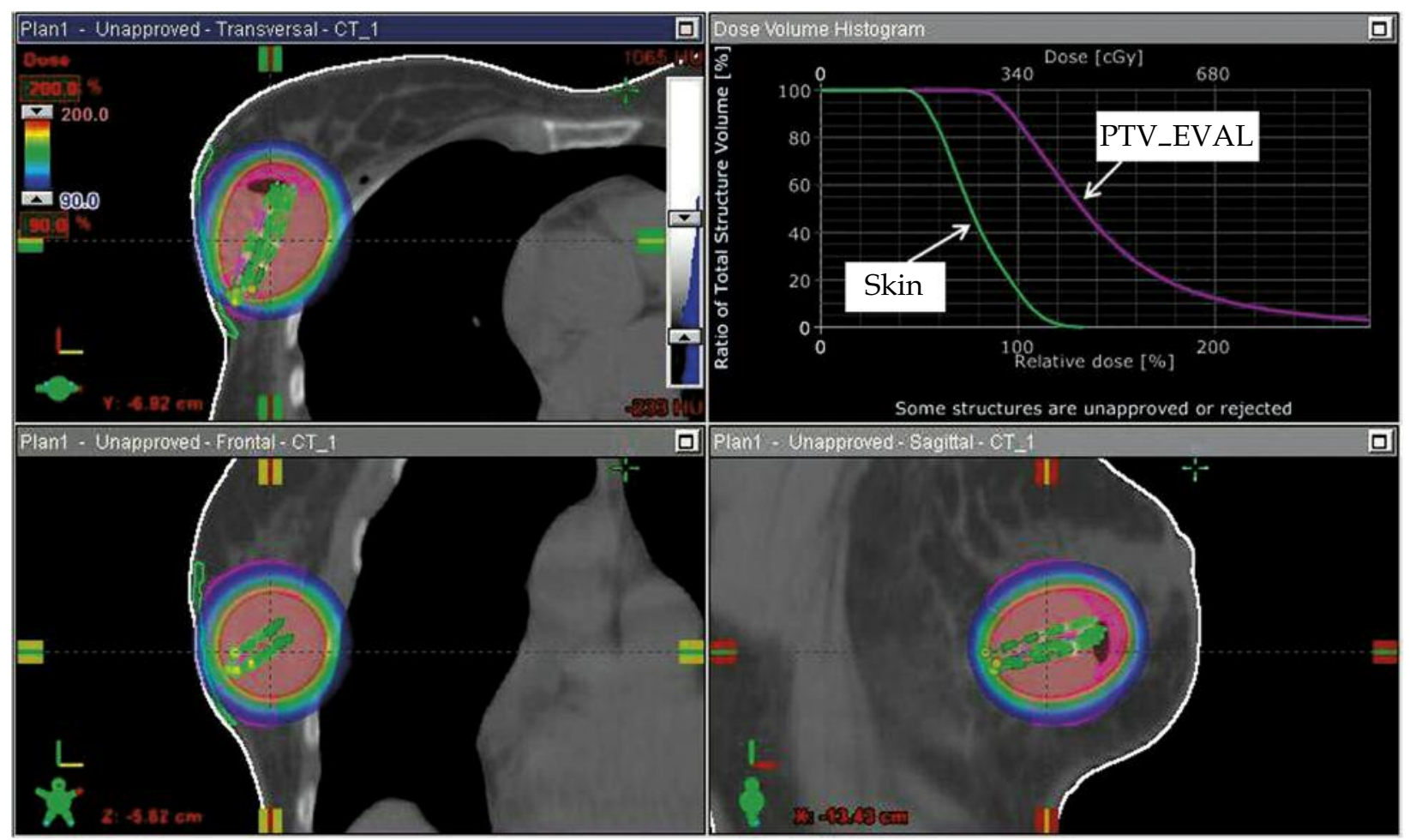

Fig. 1. Dosimetric screen capture (Varian Brachyvision ${ }^{\circledR}$, Palo Alto, CA) for a 62-year-old patient with a minimum balloon-toskin distance of $4.0 \mathrm{~mm}$. The patient was treated with a four-catheter multilumen balloon and the figure above illustrates the 90-200\% dose cloud in the axial (top left), coronal (bottom left) and sagittal (bottom right). The dose volume histogram in the top right shows the PTV_EVAL target (violet) and SKIN avoidance structure each meeting acceptable criteria

stics are summarized in Table 1 . The median age of patients treated was 56 years (range 50-81). The majority of patients had a diagnosis of infiltrating ductal carcinoma (50.0\%), followed by DCIS (38.5\%) and infiltrating lobular carcinoma $(11.5 \%)$. All patients had in situ or T1 disease. The majority of patients were estrogen and/or progesterone receptor positive $(96.2 \%)$. Only $2 / 26$ patients $(7.7 \%)$ received chemotherapy. Dosimetric characteristics are shown in Table 2. More patients had placement of the catheter with closed technique than an open cavity technique $(80.8 \%$ vs. 19.2\%). Median skin spacing was $6 \mathrm{~mm}$ (range 2.7-7). Out of the 26 patients treated, all patients had skin spacing $\leq 7 \mathrm{~mm}$ and $10 / 26$ (38.5\%) had skin spacing that was $\leq 5 \mathrm{~mm}$. Median balloon volume was $35 \mathrm{cc}$ (range 33-75). The maximum dose to the structure defined as "SKIN" (defined as a maximum point dose to $0.01 \mathrm{cc}$ of tissue) ranged between 98.5-147.4\% (median 118.2). There were no excessive hot spots as the median V150 and V200 were 23.2 and 6.1 cc, respectively.

The toxicity and cosmetic outcomes are presented in Table 3. Approximately one third of patients (34.6\%) had acute skin toxicity that qualified as grade 2 (dry desquamation) or higher and only $1 / 26$ patients experienced a skin reaction that resulted in moist desquamation. We performed a univariate analysis to identify determinants of grade 2 or higher skin effects and found no statistically significant links, specifically with regard to skin distance $(p=0.384)$ and maximum skin dose $(p=0.182)$. The majority, $25 / 26(92.3 \%)$ of the patients did not experience any infection. The patients were on prophylactic antibiotics while the catheter was in place. Most patients did not develop telangiectasias, occurring in only $7.7 \%$ of patients. Subcutaneous toxicity that was defined as visually apparent or requiring surgical intervention only occurred in merely $3.8 \%$ of patients. Only one patient $(3.8 \%)$ had severe pain which required narcotic pain medications or surgical intervention. The majority of patients had a good/excellent cosmetic outcome using the Harvard criteria $(92.3 \%)$.

\section{Discussion}

We reviewed our experience with 26 patients that underwent adjuvant partial breast irradiation with MLB brachytherapy with skin spacing $\leq 7 \mathrm{~mm}$ and evaluated acute toxicity, early cosmetic outcome and dosimetric parameters as the majority of previously published series did not include many of these patients. In regards to acute toxicity, cosmetic outcome and dosimetric parameters, it appears that these rates compare favorably with series in patients treated with APBI and skin spacing generally greater than that of our cohort $[5,11-13]$.

\section{Dosimetric characteristics}

MLB brachytherapy allows for dosimetric advantages compared to the original MammoSite in limiting skin doses. Due to the single central lumen of the Mammosite, 
Table 1. Patient and tumor characteristics

\begin{tabular}{|c|c|}
\hline Characteristic & $\begin{array}{l}\text { Treated patients } \\
(n=26)\end{array}$ \\
\hline Follow up (mo): median (range) & $9(3-15.8)$ \\
\hline \multicolumn{2}{|l|}{ Age (y) } \\
\hline Median (range) & $56(50-81)$ \\
\hline$\geq 60, n(\%)$ & $9(34.6)$ \\
\hline $50-60, n(\%)$ & $17(65.4)$ \\
\hline \multicolumn{2}{|l|}{ Race: $n(\%)$} \\
\hline African-American & $5(19.2)$ \\
\hline Caucasian & $21(80.8)$ \\
\hline \multicolumn{2}{|l|}{ Pathology: $n(\%)$} \\
\hline Ductal carcinoma in situ & $10(38.5)$ \\
\hline Invasive ductal carcinoma & $13(50.0)$ \\
\hline Invasive lobular carcinoma & $3(11.5)$ \\
\hline \multicolumn{2}{|l|}{ T stage: $n(\%)$} \\
\hline Tis & $10(38.5)$ \\
\hline T1a & $3(11.5)$ \\
\hline $\mathrm{T} 1 \mathrm{~b}$ & $7(26.9)$ \\
\hline $\mathrm{T1c}$ & $6(23.1)$ \\
\hline \multicolumn{2}{|l|}{ Estrogen receptor: $n(\%)$} \\
\hline Positive & $25(96.2)$ \\
\hline Negative & $1(3.8)$ \\
\hline \multicolumn{2}{|l|}{ Progesterone receptor: $n(\%)$} \\
\hline Positive & $25(96.2)$ \\
\hline Negative & $1(3.8)$ \\
\hline \multicolumn{2}{|l|}{ Chemotherapy } \\
\hline Yes & $2(7.7)$ \\
\hline No & $24(92.3)$ \\
\hline
\end{tabular}

the dose to the skin is closely linked to the skin distance. Without the additional lumens that are present in the Contura and MammoSite ML applicators, it may be difficult to manipulate dose away from key structures such as skin and chest wall in patients with limited skin spacing. To avoid increased skin doses and abandoning the procedure in this scenario, other dosimetric parameters such as target volume coverage may need to be compromised.

In a study by Cuttino et al., patients received less skin dose when treated with Contura MLB brachytherapy when compared to patients that received treatment with the original MammoSite despite having similar mean skin spacing. The difference became more clinically relevant when skin spacing was $<1 \mathrm{~cm}[8]$. In our study, all patients had skin spacing $\leq 7 \mathrm{~mm}$ and dosimetric characteristics of patients treated with a multicatheter balloon were examined. The dosimetric results in this group of patients with skin spacing $\leq 7 \mathrm{~mm}$ (range $2.7-7 \mathrm{~mm}$ ) compare very favorably to the criteria required to enroll patients on the current NSABP B-39/RTOG 0413 protocol. In all but one patient in this series, the maximum skin dose ( $<145 \%$ of PD) was met and this patient had a maximum skin dose of $147.4 \%$.
Table 2. Dosimetric and treatment related characteristics

\begin{tabular}{|c|c|}
\hline Characteristic & $n(\%)$ \\
\hline Number of patients & 26 \\
\hline \multicolumn{2}{|l|}{ Placement technique } \\
\hline Open & $5(19.2)$ \\
\hline Closed & $21(80.8)$ \\
\hline \multicolumn{2}{|l|}{ Skin spacing (mm) } \\
\hline Median (range) & $6(2.7-7)$ \\
\hline$\leq 5 \mathrm{~mm}$ & $10(38.5)$ \\
\hline$>5-7 \mathrm{~mm}$ & $16(61.5)$ \\
\hline \multicolumn{2}{|l|}{ Balloon volume (cc) } \\
\hline Median & 35 \\
\hline Range & $33-75$ \\
\hline \multicolumn{2}{|l|}{ Skin dose (\% of PD) } \\
\hline Median & 118.2 \\
\hline Range & $98.5-147.4$ \\
\hline \multicolumn{2}{|l|}{ V95 (\%) } \\
\hline Median & 95.6 \\
\hline Range & $90.0-99.1$ \\
\hline \multicolumn{2}{|l|}{ V100 (\%) } \\
\hline Median & 90.2 \\
\hline Range & $82.5-95.7$ \\
\hline \multicolumn{2}{|l|}{ V150 (cc) } \\
\hline Median & 23.2 \\
\hline Range & $12.1-36.2$ \\
\hline \multicolumn{2}{|l|}{ V200 (cc) } \\
\hline Median & 6.1 \\
\hline Range & $1.5-10$ \\
\hline
\end{tabular}

The V150/V200 ( $\leq 50 / \leq 10 \mathrm{cc})$ criteria were met in all patients. This was done without sacrificing coverage of PTV_EVAL as the median V95/V100 were $95.5 \%$ and $90.15 \%$, respectively. The NSABP B-39/RTOG 0413 protocol requires that $90 \%$ of PTV_EVAL is covered by $90 \%$ of prescribed dose which was met in all of the patients in this study. The use of MLB brachytherapy allows for the ability to meet dosimetric constraints similar to those in previously published reports and NSABP B-39/RTOG 0413 protocol, even in patients with close skin spacing. It is conceivable that even with limited skin spacing, more stringent criteria for target volume coverage could be met with MLB brachytherapy. The dosimetric endpoints seen in this cohort show that MLB brachytherapy allows for the treatment of patients with previously considered non-ideal candidates for this type of treatment modality.

\section{Acute toxicity and cosmesis}

Although not uniformly reported across all series, there are several toxicities that are reported more commonly such as rates of infection, acute skin reaction, symptomatic sero- 
Table 3. Toxicity and cosmesis

\begin{tabular}{|c|c|}
\hline \multicolumn{2}{|l|}{ Acute skin reaction } \\
\hline None & $8(30.8)$ \\
\hline Erythema & $9(34.6)$ \\
\hline Dry desquamation & $8(30.8)$ \\
\hline Moist desquamation & $1(3.8)$ \\
\hline \multicolumn{2}{|l|}{ Infection } \\
\hline None & $25(96.2)$ \\
\hline Oral antibiotics required & $0(0)$ \\
\hline Intravenous antibiotics required & $0(0)$ \\
\hline Surgical intervention & $1(3.8)$ \\
\hline \multicolumn{2}{|l|}{ Telangiectasia } \\
\hline None & $24(92.3)$ \\
\hline$<2 \mathrm{~cm}^{2}$ and scant & $2(7.7)$ \\
\hline$<2 \mathrm{~cm}^{2}$ and dense & $0(0)$ \\
\hline$>2 \mathrm{~cm}^{2}$ & $0(0)$ \\
\hline \multicolumn{2}{|l|}{ Subcutaneous toxicity } \\
\hline None & $16(61.5)$ \\
\hline Palpable changes & $9(34.6)$ \\
\hline Visually apparent & $1(3.8)$ \\
\hline Surgical intervention & $0(0.0)$ \\
\hline \multicolumn{2}{|l|}{ Pain } \\
\hline None & $20(76.9)$ \\
\hline Intermittent & $5(19.2)$ \\
\hline Persistent & $0(0.0)$ \\
\hline Medication required & $0(0.0)$ \\
\hline Surgical intervention & $1(3.8)$ \\
\hline \multicolumn{2}{|l|}{ Overall cosmesis (Harvard scale) } \\
\hline Excellent & $14(53.8)$ \\
\hline Good & $10(38.5)$ \\
\hline Fair & $2(7.7)$ \\
\hline Poor & $0(0.0)$ \\
\hline
\end{tabular}

ma formation and cosmetic outcome. In the current study, the most commonly observed acute toxicity was grade 1 or 2 dermatitis which occurred at a rate of $65.4 \%$. This is slightly higher than what was observed in larger series using the original MammoSite which reported grade 1 or 2 dermatitis rates between $37.8-48 \%$ [13,14]. Although the rates of grade 1 or 2 dermatitis were slightly higher, there was only one patient $\geq$ grade 3 acute skin toxicity. The infection rates observed were also in line with previously published reports, with a rate of $3.8 \%$ in this study with the use of prophylactic antibiotics. In the ASBS series of 1440 patients, the infection rate was reported as $9.5 \%$ [15]. The rate of symptomatic seroma formation varies in the published literature from $2.3-27.3 \%[5,11,12,16]$. The rate of a visually apparent seroma requiring drainage was low in this series with it occurring in only $3.8 \%$ of patients.

With a median follow up of 9 months, the early cosmetic outcome was excellent/good in $92.3 \%$ of patients. These re- sults are comparable to previously published reports in patients treated with greater skin spacing with a single lumen Mammosite which range from 90-96.9\% [12-14,17]. Increased skin spacing is a factor associated with good/excellent cosmetic outcome in patients treated with the single lumen Mammosite [15]. The development of MLB brachytherapy with Contura or Mammosite ML allows for the manipulation of dose to decrease maximum skin dose in patients with limited skin spacing. Decreased skin spacing as a patient characteristic predicting for poor cosmetic outcome is overcome by the ability to manipulate the dose distribution using multiple lumens resulting in good/excellent cosmetic outcomes in the majority of patients.

Acute toxicity and cosmetic outcome in our series compared favorably to series using the original Mammosite, despite all of the patients having limited skin spacing $(\leq 7 \mathrm{~mm})$ when undergoing treatment. The median skin spacing in this series was $5.8 \mathrm{~mm}$. This is in contrast to median skin spacing ranging from $9-11.7 \mathrm{~mm}$ in several single lumen Mammosite series $[12,13,17]$. Even in series evaluating MLB brachytherapy, the median skin spacing was greater than our series at $10 \mathrm{~mm}$ [11]. Other studies have shown a tight correlation between acute toxicity and the subsequent development of late toxicity such as fibrosis, telangectasias and poor cosmesis $[13,14]$. Therefore, even with early follow up, we believe our results serve as an accurate barometer of low risk for late effects and that this study shows the feasibility of treatment MLB brachytherapy in patients with limited skin spacing.

\section{Information}

This data was presented partially at the 2011 Annual Meeting of the American Brachytherapy Society in San Diego, California from April 14-16.

\section{References}

1. Smith BD, Arthur DW, Buchholz TA et al. Accelerated partial breast irradiation consensus statement from the American Society for Radiation Oncology (ASTRO). Int J Radiat Oncol Biol Phys 2009; 74: 987-1001.

2. Arthur DW, Vicini FA. Accelerated partial breast irradiation as a part of breast conservation therapy. J Clin Oncol 2005; 23: 1726-1735.

3. Polgar C, Fodor J, Major T et al. Breast-conserving treatment with partial or whole breast irradiation for low-risk invasive breast carcinoma - 5-year results of a randomized trial. Int J Radiat Oncol Biol Phys 2007; 69: 694-702.

4. Vicini FA, Antonucci JV, Wallace M et al. Long-term efficacy and patterns of failure after accelerated partial breast irradiation: a molecular assay-based clonality evaluation. Int J Radiat Oncol Biol Phys 2007; 68: 341-346.

5. Vicini F, Beitsch PD, Quiet CA et al. Three-year analysis of treatment efficacy, cosmesis, and toxicity by the American Society of Breast Surgeons MammoSite Breast Brachytherapy Registry Trial in patients treated with accelerated partial breast irradiation (APBI). Cancer 2008; 112: 758-766.

6. Arthur DW, Vicini FA, Todor DA et al. Improvements in critical dosimetric endpoints using the Contura multilumen balloon breast brachytherapy catheter to deliver accelerated partial breast irradiation: preliminary dosimetric findings of a phase iv trial. Int J Radiat Oncol Biol Phys 2011; 79: 26-33.

7. Brown S, McLaughlin M, Pope DK et al. A dosimetric comparison of the Contura multilumen balloon breast brachyther- 
apy catheter vs. the single-lumen MammoSite balloon device in patients treated with accelerated partial breast irradiation at a single institution. Brachytherapy 2010.

8. Cuttino LW, Todor D, Rosu M et al. A comparison of skin and chest wall dose delivered with multicatheter, Contura multilumen balloon, and MammoSite breast brachytherapy. Int J Radiat Oncol Biol Phys 2011; 79: 34-38.

9. Wilder RB, Curcio LD, Khanijou RK et al. A Contura catheter offers dosimetric advantages over a MammoSite catheter that increase the applicability of accelerated partial breast irradiation. Brachytherapy 2009; 8: 373-378.

10. NSABP B-39, RTOG 0413: A Randomized Phase III Study of conventional whole breast irradiation versus partial breast irradiation for women with stage 0 , I, or II breast cancer. Clin Adv Hematol Oncol 2006; 4: 719-721.

11. Brown S, McLaughlin M, Pope K et al. Initial radiation experience evaluating early tolerance and toxicities in patients undergoing accelerated partial breast irradiation using the Contura Multi-Lumen Balloon breast brachytherapy catheter. Brachytherapy 2009; 8: 227-233.

12. Chao KK, Vicini FA, Wallace $M$ et al. Analysis of treatment efficacy, cosmesis, and toxicity using the MammoSite breast brachytherapy catheter to deliver accelerated partial-breast irradiation: the William Beaumont hospital experience. Int J Radiat Oncol Biol Phys 2007; 69: 32-40.

13. Cuttino LW, Keisch M, Jenrette JM et al. Multi-institutional experience using the MammoSite radiation therapy system in the treatment of early-stage breast cancer: 2-year results. Int J Radiat Oncol Biol Phys 2008; 71: 107-114.

14. Dragun AE, Harper JL, Jenrette JM et al. Predictors of cosmetic outcome following MammoSite breast brachytherapy: a single-institution experience of 100 patients with two years of follow-up. Int J Radiat Oncol Biol Phys 2007; 68: 354-358.

15. Goyal S, Khan AJ, Vicini F et al. Factors associated with optimal cosmetic results at 36 months in patients treated with accelerated partial breast irradiation (APBI) on the American Society of Breast Surgeons (ASBrS) MammoSite Breast Brachytherapy Registry Trial. Ann Surg Oncol 2009; 16: 2450-2458.

16. Israel PZ, Robbins $A B$, Shroff $P$ et al. Initial surgical experience evaluating early tolerance and toxicities in patients undergoing accelerated partial breast irradiation using the Contura Multi Lumen Balloon breast brachytherapy catheter. Am Surg 2009; 75: 1042-1049.

17. Vicini FA, Beitsch PD, Quiet CA et al. First analysis of patient demographics, technical reproducibility, cosmesis, and early toxicity: results of the American Society of Breast Surgeons MammoSite breast brachytherapy trial. Cancer 2005; 104: 1138-1148. 